\title{
Relaxação Lagrangiana Aplicada ao Problema de Dimensionamento de Lotes em Máquinas Paralelas: Limitantes Inferiores ${ }^{1}$
}

D.J. FIOROTTO ${ }^{2}$, S.A. DE ARAUJO ${ }^{3}$, Departamento de Matemática Aplicada, IBILCE, UNESP - Univ Estadual Paulista, 15054-000 São José do Rio Preto, SP, Brasil.

\begin{abstract}
Resumo. Este trabalho aborda o problema de dimensionamento de lotes monoestágio em um ambiente com máquinas paralelas distintas. Cada item pode ser produzido em qualquer máquina e incorre em um tempo de preparação antes de começar a produção. O objetivo do trabalho consiste em obter limitantes inferiores de boa qualidade para este problema. Para tanto, é desenvolvido um método de solução baseado numa reformulação do problema e na relaxação Lagrangiana de um conjunto de restrições. Alguns resultados computacionais são apresentados comparando o método proposto com um trabalho da literatura e com um pacote computacional.
\end{abstract}

Palavras-chave. Dimensionamento de Lotes, Máquinas Paralelas, Limitantes Inferiores.

\section{Introdução}

Este artigo trata de problemas que aparecem no contexto do planejamento da produção industrial. De forma geral, o planejamento da produção considera decisões de aquisição de recursos e matérias-primas, bem como, decisões sobre as atividades de produção necessárias para transformar as matérias-primas em itens finais da maneira mais eficiente possível. Em algumas indústrias, tais decisões sobre as atividades de produção estão estreitamente relacionadas a decisões sobre os lotes de itens finais que devem ser produzidos. Nesse contexto tem-se o problema de dimensionamento de lotes que consiste, basicamente, em determinar os tamanhos de lotes de produção (isto é, quantidades a serem produzidas de cada item) em cada um dos períodos do horizonte de planejamento, de maneira a minimizar custos de produção ou maximizar margens de contribuição ao lucro e a satisfazer restrições de disponibilidade de recursos e atendimento à demanda dos itens.

O problema estudado neste trabalho envolve o planejamento da produção de múltiplos itens, em um único estágio. O setor de produção é composto de máquinas

\footnotetext{
${ }^{1}$ Este trabalho teve o apoio financeiro da CAPES, CNPq e FAPESP.

${ }^{2}$ diego fiorotto@hotmail.com.

${ }^{3}$ saraujo@ibilce.unesp.br.
} 
paralelas distintas com capacidade limitada. Os itens podem ser produzidos em qualquer uma das máquinas e para iniciar a produção de um item incorre-se um tempo de preparação da máquina utilizada.

A intenção deste trabalho é encontrar bons limites inferiores para este problema, para tanto uma reformulação é proposta e os limites inferiores são gerados com relaxação Lagrangiana. Diferente da maioria dos trabalhos que utilizam a decomposição tradicional, ou seja, por itens, utiliza-se a decomposição por períodos. A motivação para estudar esta abordagem decorre dos resultados obtidos em [6] para o problema com uma máquina. Tais resultados mostram que os limites inferiores resultantes da decomposição por períodos aplicada à reformulação são melhores que os obtidos utilizando a decomposição por itens aplicada à formulação clássica.

Cabe ressaltar a importância de se estudar e obter bons limites inferiores para estes problemas devido sua grande complexidade computacional, podendo assim, diminuir consideravelmente os tempos e as árvores de solução dos algoritmos exatos.

\section{Formulações do Problema}

Inicialmente apresenta-se a formulação clássica estudada em [10] e em seguida a reformulação proposta.

Para a formulação matemática do problema considere os seguintes dados:

$I=\{1, \ldots, n\}$ itens, $J=\{1, \ldots, r\}$ máquinas e $T=\{1, \ldots, m\}$ períodos;

$d_{i t}$ : demanda do item $i$ no período $t$;

$s d_{i t \tau}$ : soma da demanda do item $i$, do período $t$ até um período qualquer $\tau(\tau \geq t)$;

$h c_{i t}$ : custo unitário de estoque do item $i$ no período $t$;

$s c_{i j t}$ : custo de preparo do item $i$ na máquina $j$ no período $t$;

$v c_{i j t}$ : custo de produção do item $i$ na máquina $j$ no período $t$;

$f c_{i}$ : custo unitário de estoque inicial para o item $i$;

$s t_{i j t}$ : tempo de preparo do item $i$ na máquina $j$ no período $t$;

$v t_{i j t}$ : tempo de produção do item $i$ na máquina $j$ no período $t$;

$C a p_{j t}$ : capacidade (em unidade de tempo) da máquina $j$ no período $t$.

Variáveis de decisão:

$x_{i j t}$ : unidades produzidas do item $i$ na máquina $j$ no período $t$;

$y_{i j t}$ : variável binária, indicando a produção ou não do item $i$ na máquina $j$ no período $t$;

$s_{i t}$ : estoque do item $i$ no final do período $t$;

$s_{i 0}$ : quantidade de estoque inicial para o item $i$.

Formulação matemática:

$$
\operatorname{Min} \sum_{i=1}^{n} f c_{i} s_{i 0}+\sum_{i=1}^{n} \sum_{j=1}^{r} \sum_{t=1}^{m}\left(s c_{i j t} y_{i j t}+v c_{i j t} x_{i j t}\right)+\sum_{i=1}^{n} \sum_{t=1}^{m} h c_{i t} s_{i t}
$$


Sujeito a:

$$
\begin{array}{lr}
s_{i, t-1}+\sum_{j=1}^{r} x_{i j t}=d_{i t}+s_{i t} & \forall i \in I, t \in T \\
x_{i j t} \leq s d_{i t m} y_{i j t} & \forall i \in I, j \in J, t \in T \\
\sum_{i=1}^{n}\left(s t_{i j t} y_{i j t}+v t_{i j t} x_{i j t}\right) \leq C a p_{j t} & \forall j \in J, t \in T \\
y_{i j t} \in\{0,1\}, x_{i j t} \geq 0, s_{i t} \geq 0, s_{i 0} \geq 0, s_{i m}=0 & \forall i \in I, j \in J, t \in T .
\end{array}
$$

A função objetivo (2.1) minimiza os custos totais de preparação, produção, estoque e estoque inicial. As restrições (2.2) garantem o balanceamento de estoque. Para evitar problemas infactíveis o modelo considera a possibilidade de estoque inicial, contudo o custo $f c_{i}$ para este estoque é muito grande, assim $s_{i 0}$ terá valor diferente de zero apenas quando o problema não tiver solução factível. Em seguida, tem-se as restrições de preparação de máquina (2.3) e as limitações de capacidade (2.4). Finalmente, em (2.5) tem-se a definição dos domínios das variáveis.

Este problema pode ser reformulado usando a abordagem de redefinição das variáveis proposta em [5], originando uma formulação baseada no problema de caminho mínimo. Cada nó do grafo representa um período, incluindo um período falso $m+1$. Existe um arco entre cada par de nós e o arco entre os nós $t$ e $q(q>t)$ representa a opção de produção da soma da demanda entre o período $t$ e o período $q-1$ no período $t$. O valor de cada arco é dado pelo custo da respectiva opção de produção. O objetivo é encontrar o menor caminho de 1 até $m+1$. Em [5] os autores consideraram o problema sem restrição de capacidade e em [6] a ideia foi estendida para o caso em que se tem restrições de capacidade.

Para a reformulação defina os seguintes parâmetros:

$c v_{i j t k}$ : custo de produção e estoque para produzir o item $i$, na máquina $j$ no período $t$ atendendo a demanda dos períodos $t$ até $k$

$$
=v c_{i j t} s d_{i t k}+\sum_{s=t+1}^{k} \sum_{u=t}^{s-1} h c_{i u} d_{i s} ;
$$

$c i_{i t}$ : custo para que o estoque inicial do item $i$ atenda a demanda dos períodos 1 até o período $t$

$$
=f c_{i} s d_{i 1 t}+\sum_{s=2}^{t} \sum_{u=1}^{s-1} h c_{i u} d_{i s} .
$$

Tem-se também as seguintes novas variáveis para o modelo:

$z v_{i j t k}$ : fração do plano de produção do item $i$ na máquina $j$, em que a produção no período $t$ atende a demanda do período $t$ até o período $k$;

$w_{i t}$ : fração do plano de estoque inicial do item $i$ em que a demanda é atendida para os primeiros $t$ períodos.

A reformulação baseada no problema do caminho mínimo é a seguinte:

$$
\operatorname{Min} \sum_{i=1}^{n} \sum_{t=1}^{m} c i_{i t} w_{i t}+\sum_{i=1}^{n} \sum_{j=1}^{r} \sum_{t=1}^{m} s c_{i j t} y_{i j t}+\sum_{i=1}^{n} \sum_{j=1}^{r} \sum_{t=1}^{m} \sum_{k=t}^{m} c v_{i j t k} z v_{i j t k}
$$


Sujeito a :

$$
\begin{array}{lr}
1=\sum_{k=1}^{m} w_{i k}+\sum_{j=1}^{r} \sum_{k=1}^{m} z v_{i j 1 k} & \forall i \in I \quad \text { (2.7) } \\
w_{i, t-1}+\sum_{j=1}^{r} \sum_{k=1}^{t-1} z v_{i, j, k, t-1}=\sum_{j=1}^{r} \sum_{k=t}^{m} z v_{i j t k} & \forall i \in I, t \in T /\{1\} \quad \text { (2.8) } \\
\sum_{k=t}^{m} z v_{i j t k} \leq y_{i j t} & \forall i \in I, j \in J, t \in T \quad(2.9) \\
\sum_{i=1}^{n} s t_{i j t} y_{i j t}+\sum_{i=1}^{n} \sum_{k=t}^{m} v t_{i j t} s d_{i t k} z v_{i j t k} \leq C a p_{j t} & \forall i \in I, j \in J, t \in T(2.10) \\
y_{i j t} \in\{0,1\}, \quad w_{i t} \geq 0 & \forall i \in I, j \in J(2.11) \\
z v_{i j t k} \geq 0 & \forall i \in I, j \in J, t \in T \quad \forall k \in T, k \geq t .(2.12)
\end{array}
$$$$
\forall i \in I, j \in J(2.11)
$$

A função objetivo (2.6) minimiza a soma dos custos de estoque inicial, preparação, produção e estocagem. As restrições (2.7) e (2.8) definem as restrições de fluxo para a rede de caminho mínimo. Para cada item, uma unidade de fluxo é enviada à rede, impondo que a demanda de cada produto seja satisfeita sem atraso. As restrições (2.9) forçam a preparação para cada item a ser produzido. As restrições de capacidade (2.10) limitam a soma total dos tempos de preparação e produção pela capacidade disponível em cada período e em cada máquina. As restrições (2.11) e (2.12) definem o domínio das variáveis.

Para este modelo, devido às restrições de capacidade, as variáveis $w_{i t}$ e $z v_{i j t k}$ podem ter valores fracionários. A interpretação para um valor fracionário, por exemplo, 0.3 para uma variável $z v_{i j t k}$ é a seguinte: produzir no período $t$, na máquina $j$, $30 \%$ do total da demanda do período $t$ até o período $k$.

\section{Relaxação Lagrangiana Aplicada às Restrições de Fluxo}

Nesta seção apresenta-se uma relaxação Lagrangiana baseada em [6], em que considerase o modelo (2.6) - (2.12) e as restrições de fluxo (2.7) e (2.8) são dualizadas na função objetivo. O problema se decompõe em subproblemas independentes, por períodos e por máquinas e contém as restrições de capacidade, preparação e as condições de integralidade.

Para o problema com uma única máquina, além de [6], os trabalhos [4] e [7] testam a decomposição por períodos considerando a formulação clássica. De seus testes computacionais, concluíram que, quando aplicada à formulação clássica, a decomposição por itens é superior à decomposição por períodos. Em [2] provase que, para o problema com uma única máquina e sem tempos de preparação, o limitante inferior obtido pela decomposição por períodos para a reformulação é melhor que o obtido pela decomposição por itens da formulação clássica. Em [8] é testada a decomposição por períodos aplicada a uma reformulação do problema com 
uma única máquina e sem custos de preparação. Os autores chegam à conclusão de que, para a reformulação, a decomposição por períodos é superior à decomposição por itens. Deve-se observar que não encontramos na literatura nenhum trabalho que considera tal reformulação e a decomposição por períodos para o caso de máquinas paralelas.

Na relaxação Lagrangiana as restrições $(2.7)$ e (2.8) são dualizadas na função objetivo (2.6) com multiplicadores Lagrangianos $p_{i t}$.

$$
\begin{gathered}
\text { Min } \sum_{i=1}^{n} \sum_{t=1}^{m} c i_{i t} w_{i t}+\sum_{i=1}^{n} \sum_{j=1}^{r} \sum_{t=1}^{m} s c_{i j t} y_{i j t}+\sum_{i=1}^{n} \sum_{j=1}^{r} \sum_{t=1}^{m} \sum_{k=t}^{m} c v_{i j t k} z v_{i j t k}- \\
-\sum_{i=1}^{n} p_{i 1}\left(\sum_{k=1}^{m} w_{i k}+\sum_{j=1}^{r} \sum_{k=1}^{m} z v_{i j 1 k}-1\right)- \\
-\sum_{i=1}^{n} \sum_{t=2}^{m} p_{i t}\left(\sum_{j=1}^{r} \sum_{k=t}^{m} z v_{i j t k}-w_{i, t-1}-\sum_{j=1}^{r} \sum_{k=1}^{t-1} z v_{i j k, t-1}\right)
\end{gathered}
$$

Após reorganizar os termos da função objetivo, o problema Lagrangiano tornase:

$$
\begin{aligned}
\operatorname{Min} \sum_{i=1}^{n} \sum_{j=1}^{r} \sum_{t=1}^{m} s c_{i j t} y_{i j t}+ & \sum_{i=1}^{n} \sum_{t=1}^{m-1}\left(c i_{i t}-p_{i, 1}+p_{i, t+1}\right) w_{i t}+ \\
+ & \sum_{i=1}^{n}\left(c i_{i m}-p_{i, 1}\right) w_{i m}+\sum_{i=1}^{n} \sum_{j=1}^{r} \sum_{t=1}^{m} \sum_{k=t}^{m-1}\left(c v_{i j t k}-p_{i t}+p_{i, k+1}\right) z v_{i j t k}+ \\
& +\sum_{i=1}^{n} \sum_{j=1}^{r} \sum_{t=1}^{m}\left(c v_{i j t m}-p_{i t}\right) z v_{i j t m}+\sum_{i=1}^{n} p_{i 1}
\end{aligned}
$$

Sujeito a : (2.9)-(2.12)

O problema Lagrangiano pode ser decomposto em subproblemas independentes para cada período $t$ e cada máquina $j$. Estes subproblemas podem ser resolvidos pelo método branch-and-bound proposto por [6]. Os multiplicadores Lagrangianos $p_{i t}$ são atualizados pelo método de otimização do subgradiente ([1]).

Para iniciar o método, fixamos as variáveis duais em zero $\left(p_{i t}^{0}=0\right)$ e o tamanho do passo na direção do subgradiente é proporcional a um parâmetro $\alpha$, que deve decrescer com o número de iterações. A regra utilizada para gerar uma sequência decrescente para $\alpha$ é caracterizada pelos parâmetros $\left(\alpha_{0}, r_{0}, d\right)$. Os valores de $\alpha$ são obtidos fazendo $\alpha=\alpha_{0}$ e é diminuído progressivamente multiplicando-o por um parâmetro $d$, se a solução Lagrangiana não for melhorada nas últimas $r=r_{0}$ iterações. Após extensivos testes computacionais, com o objetivo de definir a melhor combinação desses parâmetros, os valores definidos são $\alpha_{0}=1, r_{0}=50, d=0.6 \mathrm{e}$ são realizadas 2500 iterações do método.

Ainda para o método do subgradiente, foi desenvolvida uma heurística para a obtenção de um limite superior (solução factível) utilizado na atualização do 
tamanho do passo. Para tanto, as ideias apresentadas em [9] foram estendidas e adaptadas para o problema em questão. A heurística consiste nos seguintes passos:

1. Para uma dada iteração, utilizar o plano de preparação (variáveis $y_{i j t}$ ) que é obtido ao resolver os subproblemas, indicando se há ou não preparação de determinado item $i$ na máquina $j$ em um período $t$. Observe que este plano será factível no que diz respeito às restrições de capacidade.

2. Com as variáveis $y_{i j t}$ fixadas, resolver o problema de fluxo de redes para a primeira máquina.

3. Subtrair da demanda total a demanda satisfeita com a primeira máquina e resolver um novo problema de fluxo de redes para a próxima máquina. Repetir este procedimento até a última máquina.

4. Para a última máquina somar ao valor total as quantidades de produção, estoque inicial (se necessário) e estoque. Este valor por sua vez gera uma solução factível (limite superior) para o problema original.

\section{Resultados Computacionais}

O procedimento descrito na seção anterior, denotado $F A$, foi testado em um total de 2880 instâncias propostas em [10]. Foram feitas comparações com o pacote CPLEX 12.2 e com o método proposto por Toledo e Armentano [10], denotado aqui por $T A$. Todos os testes foram realizados em um microcomputador AMD Turion $1.8 \mathrm{GHz}$ com 3 GB de memória RAM e sistema operacional Windows XP.

As 2880 instâncias são divididas em 8 tipos diferentes de classes que são geradas com valores altos e baixos para os custos de preparação (SA ou SB), para tempos de preparação (TA ou TB) e com capacidades normal e apertada (CN ou CA). Portanto, a classe CNSBTB, por exemplo, refere-se a instâncias com capacidade normal, custos de preparação baixos e tempos de preparação baixos. A notação para as demais classes seguem esse mesmo raciocínio. Ainda, são geradas 10 instâncias para cada classe e configuração de número de máquinas $(r=2,4$ e 6$)$, ítens $(n=$ $6,12,25$ e 50$)$ e períodos $(m=6,12$ e 18$)$.

Os parâmetros foram gerados em intervalos $[a, b]$ com distribuição uniforme e denotado $U[a, b]$ :

- custo de produção $\left(v c_{i j}\right) \mathrm{U}[1.5,2.5]$;

- custo de preparação $\left(s c_{i j}\right) \mathrm{U}[5.0,95.0]$;

- custo de estoque $\left(h c_{i}\right) \mathrm{U}[0.2,0.4]$;

- tempo de produção $\left(v t_{i j}\right) \mathrm{U}[1.0,5.0]$;

- tempo de preparação $\left(s t_{i j}\right) \mathrm{U}[10.0,50.0] ;$

- demanda $\left(d_{i t}\right) \mathrm{U}[0,180]$. 
Para gerar exemplares com custos de preparação alto multiplicam-se os custos gerados por 10. Da mesma forma, para gerar exemplares com tempos de preparação alto multiplicam-se estes por 1.5 .

A capacidade também foi gerada de acordo com [10], ou seja, toma-se como base a capacidade necessária para produzir os itens de acordo com a política lote-porlote. Posteriormente aplica-se um ajuste para reduzir a capacidade a fim de gerar instâncias com a utilização da capacidade em torno de $80 \%$. A capacidade apertada é obtida multiplicando a capacidade gerada por 0.9 .

Para avaliar a qualidade dos limites inferiores os Gaps serão calculados da seguinte forma:

$$
G a p=\frac{z_{C P L E X}-z_{L I}}{z_{L I}} * 100,
$$

onde

$z_{C P L E X}$ é o valor da solução encontrada pelo CPLEX;

$z_{L I}$ limite inferior obtido pelo próprio CPLEX ou pelos métodos $T A$ e $F A$;

Observe que utiliza-se o limite superior obtido pelo CPLEX pois o foco do trabalho está na qualidade dos limitantes inferiores. Poderíamos também fazer uma comparação entre os limitantes superiores obtidos. No entanto, no atual momento da pesquisa, os limitantes obtidos pelo método proposto não são de boa qualidade.

A Tabela 1 apresenta a quantidade de instâncias para cada configuração, número de períodos, máquinas e itens que o CPLEX 12.2 provou a otimalidade no tempo máximo de dois minutos para cada instância. Com isso pode-se analisar a dificuldade em se obter soluções para os problemas conforme suas características. Observa-se que para problemas com 6 períodos o CPLEX provou a otimalidade para quase todas as instâncias, por outro lado, para os problemas com 18 períodos, na maioria dos casos o solver não provou a otimalidade em nenhum dos exemplos. Verifica-se ainda um aumento considerável na dificuldade dos problemas quando se aumentam os custos de preparação.

Por conta do grande número de instâncias que o CPLEX provou a otimilidade para os problemas com 6 e 12 períodos, apresentaremos nas comparações entre os limites inferiores apenas as configurações com 18 períodos. Contudo, cabe observar que para todas as instâncias com 6 e 12 períodos o método proposto $(F A)$ obteve gaps em média $39 \%$ melhores que o método $T A$.

Na Tabela 2 comparam-se os limites inferiores encontrados com o CPLEX (no nó raiz depois de aplicar os cortes (coluna cortes) e após as ramificações (coluna ramif.)), com os gerados pelos métodos $T A$ e $F A$ para os problemas com 18 períodos e capacidade normal.

Os gaps calculados para o CPLEX utilizam como limites inferiores os encontrados no nó raiz após a aplicação dos cortes. Os resultados evidenciam que à medida que o número de itens aumenta, os gaps diminuem de forma significativa (sempre menores que $1 \%$ para 25 e 50 ítens). O aumento no número de máquinas faz com que os gaps também aumentem. Os custos de preparação altos causam um aumento significativo no gap. No entanto, os tempos de preparação não afetam os gaps de forma tão significativa.

Observe que, apesar da razoável diferença entre os tempos computacionais (colunas $\mathrm{T}(\mathrm{s})$ ), o método proposto $F A$ encontrou, em praticamente todas as instâncias, 


\begin{tabular}{|c|c|c|c|c|c|c|c|c|c|c|}
\hline $\mathrm{m}$ & $\mathrm{r}$ & $\mathrm{n}$ & CNSBTB & CNSATB & CNSBTA & $\overline{\text { CNSATA }}$ & CASBTB & CASATB & CASBTA & CASATA \\
\hline \multirow{12}{*}{6} & & 6 & 10 & 10 & 10 & 10 & 10 & 10 & 10 & 10 \\
\hline & 2 & 12 & 10 & 10 & 10 & 10 & 10 & 10 & 10 & 10 \\
\hline & & 25 & 10 & 10 & 10 & 10 & 10 & 10 & 10 & 9 \\
\hline & & 50 & 10 & 8 & 10 & 7 & 10 & 3 & 10 & 2 \\
\hline & & 6 & 10 & 10 & 10 & 10 & 10 & 10 & 10 & 10 \\
\hline & 4 & 12 & 10 & 10 & 10 & 10 & 10 & 8 & 10 & 9 \\
\hline & & 25 & 10 & 8 & 10 & 8 & 10 & 5 & 10 & 4 \\
\hline & & 50 & 10 & 3 & 10 & 4 & 10 & 0 & 9 & 0 \\
\hline & & 6 & 10 & 9 & 10 & 8 & 10 & 9 & 10 & 8 \\
\hline & 6 & 12 & 10 & 7 & 10 & 6 & 10 & 5 & 10 & 5 \\
\hline & & 25 & 10 & 8 & 10 & 7 & 10 & 4 & 10 & 4 \\
\hline & & 50 & 10 & 3 & 10 & 4 & 10 & 1 & 10 & 1 \\
\hline \multirow{12}{*}{12} & & 6 & 10 & 8 & 10 & 6 & 10 & 5 & 9 & 5 \\
\hline & 2 & 12 & 10 & 6 & 9 & 5 & 9 & 5 & 8 & 5 \\
\hline & & 25 & 9 & 3 & 9 & 0 & 8 & 0 & 7 & 0 \\
\hline & & 50 & 10 & 0 & 10 & 0 & 7 & 0 & 7 & 0 \\
\hline & & 6 & 8 & 0 & 8 & 0 & 7 & $\frac{1}{1}$ & 5 & 0 \\
\hline & 4 & 12 & 8 & 2 & 8 & 2 & 6 & 3 & 7 & 2 \\
\hline & & 25 & 10 & 1 & 10 & 2 & 8 & 0 & 7 & 0 \\
\hline & & 50 & 10 & 1 & 10 & 1 & 8 & 1 & 7 & 0 \\
\hline & & 6 & 5 & $\overline{0}$ & 3 & $\overline{0}$ & 1 & 0 & 1 & $\overline{0}$ \\
\hline & 6 & 12 & 8 & 0 & 7 & 0 & 6 & 0 & 6 & 0 \\
\hline & & 25 & 10 & 0 & 9 & 0 & 6 & 0 & 8 & 0 \\
\hline & & 50 & 10 & 0 & 10 & 0 & 9 & 0 & 10 & 0 \\
\hline \multirow{12}{*}{18} & & 6 & 8 & 1 & 7 & 1 & 6 & 1 & 7 & 1 \\
\hline & 2 & 12 & 8 & 2 & 7 & 2 & 6 & 0 & 3 & 1 \\
\hline & & 25 & 5 & 0 & 5 & 0 & 3 & 0 & 3 & 0 \\
\hline & & 50 & 9 & 0 & 8 & 0 & 6 & 0 & 6 & 0 \\
\hline & & 6 & 1 & 0 & 0 & 0 & 0 & 0 & 0 & 0 \\
\hline & 4 & 12 & 4 & 1 & 3 & 0 & 1 & 0 & 0 & 0 \\
\hline & & 25 & 7 & 0 & 5 & 0 & 4 & 0 & 3 & 0 \\
\hline & & 50 & 10 & 0 & 8 & 0 & 6 & 0 & 6 & 0 \\
\hline & & 6 & 0 & 0 & 0 & 0 & 0 & 0 & 0 & 0 \\
\hline & 6 & 12 & 5 & 0 & 4 & 0 & 3 & 0 & 3 & 0 \\
\hline & & 25 & 8 & 0 & 7 & 0 & 4 & 0 & 4 & 0 \\
\hline & & 50 & 10 & 0 & 9 & 0 & 8 & 0 & 6 & 0 \\
\hline
\end{tabular}

Tabela 1: Número de soluções ótimas encontradas pelo CPLEX

gaps melhores que o método $T A$. A notável diferença dos tempos computacionais entre os dois métodos explica-se pela dificuldade dos subproblemas. Enquanto que para o método $T A$ os subproblemas são problemas de dimensionamento de lotes com único item em máquinas paralelas sem restrição de capacidade, que são baratos computacionalmente, para o método $F A$, os subproblemas são problemas da mochila que são caros computacionalmente. Além disso, o método $F A$ necessita de um número maior de iterações do método do subgradiente para convergir.

Pode-se observar ainda que enquanto o método $T A$ obtém resultados melhores que o CPLEX em apenas $6 \%$ das instâncias, o proposto $F A$ supera o solver em $81 \%$, o que comprova a qualidade dos limites inferiores deste método.

A Tabela 2 mostra ainda, que mesmo após as ramificações do CPLEX os limites inferiores encontrados com o método $F A$ são melhores na maioria das instâncias com 25 e 50 itens principalmente para as classes com custos de preparação altos (CNSATB e CNSATA). Portanto, se utilizarmos os limitantes inferiores gerados pelo método $F A$ no nó raiz do CPLEX, além de obtermos gaps melhores, ajudaríamos o solver a fazer várias podas nas árvores de solução e a encontrar soluções factíveis melhores.

A Tabela 3 ilustra os resultados obtidos para as mesmas classes considerando a capacidade apertada. Pode-se observar que os gaps apresentam um aumento se comparados com a Tabela 2 (com capacidade normal) e as diferenças entre a qualidade dos limitantes tornam-se mais evidentes deixando ainda mais claro o sucesso obtido pela abordagem proposta, na busca por limitantes inferiores de melhor qualidade que os existentes na literatura. 


\begin{tabular}{|c|c|c|c|c|c|c|c|c|c|c|c|}
\hline & & & $\overline{\mathrm{CPLI}}$ & $(\mathrm{LI})$ & $\overline{\mathrm{T}}$ & & $\overline{\mathrm{F}}$ & & & Gaps & \\
\hline & $\mathrm{r}$ & $\mathrm{n}$ & cortes & ramif. & $\overline{\mathrm{LI}}$ & $\mathrm{T}(\mathrm{s})$ & LI & $\mathrm{T}(\mathrm{s})$ & cplex & TA & FA \\
\hline \multirow{12}{*}{ CNSBTB } & \multirow{4}{*}{2} & 6 & 21928 & 22024 & 21906 & 0,19 & 21972 & 0,84 & 0,50 & 0,61 & 0,30 \\
\hline & & 12 & 42494 & 43051 & 42979 & 0,41 & 43034 & 1,83 & 1,34 & 0,20 & 0,07 \\
\hline & & 25 & 89204 & 89226 & 89199 & 0,74 & 89219 & 4,19 & 0,04 & 0,05 & 0,02 \\
\hline & & 50 & 176489 & 176493 & 176479 & 1,36 & 176461 & 6,90 & 0,00 & 0,01 & 0,02 \\
\hline & \multirow{4}{*}{4} & 6 & 20096 & 20276 & 20022 & 0,56 & 20238 & 1,36 & 1,49 & 1,86 & 0,77 \\
\hline & & 12 & 39822 & 39905 & 39805 & 0,87 & 39891 & 2,59 & 0,32 & 0,36 & 0,14 \\
\hline & & 25 & 80842 & 80859 & 80837 & 1,68 & 80862 & 4,99 & 0,05 & 0,05 & 0,02 \\
\hline & & 50 & 162311 & 162315 & 162309 & 3,23 & 162318 & 9,58 & 0,01 & 0,01 & 0,00 \\
\hline & \multirow{4}{*}{6} & 6 & 19593 & 19792 & 19409 & 0,97 & 19775 & 1,85 & 2,17 & 3,14 & 1,23 \\
\hline & & 12 & 38335 & 38400 & 38314 & 1,58 & 38393 & 3,30 & 0,30 & 0,35 & 0,14 \\
\hline & & 25 & 78744 & 78759 & 78735 & 2,74 & 78753 & 6,57 & 0,05 & 0,48 & 0,04 \\
\hline & & 50 & 154664 & 154664 & 154660 & 5,28 & 154678 & 12,47 & 0,01 & 0,04 & 0,00 \\
\hline \multirow{12}{*}{ CNSATB } & \multirow{4}{*}{2} & 6 & 33582 & 34491 & 33111 & 0,35 & 33152 & 0,91 & 6,07 & 7,58 & 6,94 \\
\hline & & 12 & 65356 & 65812 & 65291 & 0,55 & 65687 & 2,14 & 1,11 & 1,21 & 0,60 \\
\hline & & 25 & 135586 & 135739 & 135641 & 1,14 & 135861 & 6,67 & 0,42 & 0,38 & 0,22 \\
\hline & & 50 & 266756 & 266803 & 266772 & 2,53 & 266953 & 16,09 & 0,12 & 0,11 & 0,05 \\
\hline & \multirow{4}{*}{4} & 6 & 29363 & 30138 & 28672 & 0,66 & 28842 & 1,52 & 10,09 & 12,74 & 10,77 \\
\hline & & 12 & 57627 & 58005 & 57435 & 1,15 & 57725 & 2,94 & 2,76 & 3,10 & 2,59 \\
\hline & & 25 & 116675 & 116808 & 116669 & 2,12 & 116948 & 6,47 & 0,45 & 0,46 & 0,22 \\
\hline & & 50 & 234998 & 235036 & 234953 & 4,25 & 235150 & 14,50 & 0,13 & 0,15 & 0,06 \\
\hline & \multirow{4}{*}{6} & 6 & 28923 & 29893 & 28145 & 1,29 & 28642 & 2,21 & 14,83 & 18,01 & 13,76 \\
\hline & & 12 & 54574 & 54837 & 54343 & 2,02 & 54967 & 3,73 & 3,45 & 3,89 & 2,71 \\
\hline & & 25 & 112340 & 112432 & 112245 & 3,70 & 112641 & 8,27 & 0,76 & 0,85 & 0,49 \\
\hline & & 50 & 218904 & 218940 & 218897 & 6,78 & 219053 & 15,54 & 0,11 & 0,12 & 0,05 \\
\hline \multirow{12}{*}{ CNSBTA } & \multirow{4}{*}{2} & 6 & 21987 & 22062 & 21925 & 0,21 & 21989 & 0,91 & 0,42 & 0,71 & 0,41 \\
\hline & & 12 & 43028 & 43090 & 42957 & 0,41 & 43074 & 4,44 & 0,19 & 0,35 & 0,08 \\
\hline & & 25 & 89240 & 89265 & 89236 & 0,80 & 89261 & 12,34 & 0,04 & 0,05 & 0,02 \\
\hline & & 50 & 176525 & 176531 & 176519 & 1,66 & 176510 & 20,10 & 0,01 & 0,01 & 0,02 \\
\hline & \multirow{4}{*}{4} & 6 & 20132 & 20315 & 20045 & 0,56 & 20278 & 1,40 & 1,54 & 1,98 & 0,80 \\
\hline & & 12 & 39843 & 39933 & 39826 & 0,92 & 39923 & 2,65 & 0,37 & 0,41 & 0,17 \\
\hline & & 25 & 80858 & 80887 & 80854 & 1,65 & 80879 & 5,10 & 0,05 & 0,06 & 0,03 \\
\hline & & 50 & 162323 & 162332 & 162320 & 3,37 & 162332 & 10,30 & 0,01 & 0,01 & 0,00 \\
\hline & \multirow{4}{*}{6} & 6 & 19620 & 19830 & 19421 & 0,91 & 19813 & 1,91 & 2,44 & 3,49 & 01,44 \\
\hline & & 12 & 38347 & 38417 & 38326 & 1,60 & 38412 & 3,35 & 0,33 & 0,38 & 0,16 \\
\hline & & 25 & 78755 & 78783 & 78747 & 2,83 & 78773 & 6,82 & 0,06 & 0,07 & 0,03 \\
\hline & & 50 & 154671 & 154677 & 154666 & 5,55 & 154686 & 12,94 & 0,01 & 0,01 & 0,00 \\
\hline \multirow{12}{*}{ CNSATA } & \multirow{4}{*}{2} & 6 & 33704 & 34582 & 33052 & 0,36 & 33217 & 0,96 & 6,64 & 8,75 & 8,21 \\
\hline & & 12 & 65555 & 66033 & 65211 & 0,59 & 65934 & 2,33 & 1,15 & 1,68 & 0,57 \\
\hline & & 25 & 135964 & 136120 & 136028 & 1,20 & 136275 & 6,94 & 0,44 & 0,40 & 0,21 \\
\hline & & 50 & 267498 & 267544 & 267502 & 2,89 & 267719 & 17,97 & 0,17 & 0,17 & 0,09 \\
\hline & \multirow{4}{*}{4} & 6 & 29446 & 30301 & 28590 & 0,69 & 28675 & 1,56 & 10,70 & 14,01 & 13,70 \\
\hline & & 12 & 57718 & 58081 & 57331 & 1,21 & 57650 & 3,06 & 2,75 & 3,45 & 2,87 \\
\hline & & 25 & 116794 & 116929 & 116796 & 2,18 & 117077 & 6,79 & 0,47 & 0,47 & 0,23 \\
\hline & & 50 & 235303 & 235342 & 235190 & 4,47 & 235394 & 15,01 & 0,13 & 0,17 & 0,09 \\
\hline & \multirow{4}{*}{6} & 6 & 29021 & 30044 & 27828 & 1,21 & 28365 & 2,23 & 17,56 & 22,60 & 20,30 \\
\hline & & 12 & 54636 & 54888 & 54393 & 2,06 & 55020 & 3,82 & 3,59 & 4,05 & 2,87 \\
\hline & & 25 & 112431 & 112520 & 112330 & 3,71 & 112735 & 8,43 & 0,78 & 0,88 & 0,51 \\
\hline & & 50 & 219012 & 219050 & 219011 & 6,93 & 219170 & 16,27 & 0,12 & 0,12 & 0,05 \\
\hline
\end{tabular}

Tabela 2: Comparação entre limites inferiores para 18 períodos e capacidade normal

\section{Conclusões e Propostas Futuras}

Neste trabalho foi estudado um problema de dimensionamento de lotes com restrições de capacidade em máquinas paralelas. O trabalho foi inspirado em [6], em 


\begin{tabular}{|c|c|c|c|c|c|c|c|c|c|c|c|}
\hline & & & CPLl & $\mathrm{K}(\mathrm{LI})$ & $\overline{\mathrm{T}}$ & & & & & Gaps & \\
\hline & $\mathrm{r}$ & $\mathrm{n}$ & cortes & ramif. & LI & $\mathrm{T}(\mathrm{s})$ & LI & $\mathrm{T}(\mathrm{s})$ & cplex & TA & FA \\
\hline & & 6 & 22017 & 22157 & 21929 & 0,25 & 22088 & 0,94 & 0,80 & 1,21 & 0,48 \\
\hline & 2 & 12 & 43128 & 43209 & 42925 & 0,50 & 43173 & 2,04 & 0,24 & 0,72 & 0,14 \\
\hline & & 25 & 89358 & 89391 & 89356 & 1,00 & 89381 & 5,05 & 0,07 & 0,07 & 0,05 \\
\hline & & 50 & 176644 & 176654 & 176642 & 1,94 & 176608 & 9,71 & 0,02 & 0,02 & 0,04 \\
\hline & & 6 & 20211 & 20402 & 20100 & 0,57 & 20373 & 1,45 & 1,88 & 2,44 & 1,07 \\
\hline CASBTB & 4 & 12 & 39922 & 40026 & 39899 & 1,06 & 40028 & 2,76 & 0,51 & 0,57 & 0,24 \\
\hline & & 25 & 80917 & 80950 & 80912 & 1,83 & 80945 & 5,41 & 0,07 & 0,08 & 0,04 \\
\hline & & 50 & 162376 & 162386 & 162374 & 3,94 & 162378 & 11,31 & 0,02 & 0,02 & 0,02 \\
\hline & & 6 & 19698 & 19924 & 19461 & 1,04 & 19906 & 2,09 & 2,83 & 4,08 & 1,75 \\
\hline & 6 & 12 & 38394 & 38474 & 38365 & 1,76 & 38479 & 3,48 & 0,38 & 0,46 & 0,16 \\
\hline & & 25 & 78802 & 78837 & 78789 & 3,22 & 78830 & 6,97 & 0,10 & 0,11 & 0,06 \\
\hline & & 50 & 154699 & 154711 & 154695 & 5,07 & 154719 & 13,37 & 0,01 & 0,02 & 0,00 \\
\hline & & 6 & 34208 & 35180 & 33162 & 0,43 & 34099 & 1,09 & 8,26 & 11,67 & 8,60 \\
\hline & 2 & 12 & 66199 & 66689 & 65735 & 0,80 & 66695 & 2,70 & 1,86 & 2,58 & 1,10 \\
\hline & & 25 & 137216 & 137370 & 137249 & 1,70 & 137556 & 8,34 & 0,68 & 0,65 & 0,43 \\
\hline & & 50 & 269829 & 269861 & 269851 & 3,86 & 269993 & 25,08 & 0,23 & 0,23 & 0,17 \\
\hline & & 6 & 29716 & 30671 & 28713 & 0,73 & 29128 & 1,63 & 12,89 & 16,83 & 15,20 \\
\hline CASATB & 4 & 12 & 58070 & 58468 & 57806 & 1,37 & 57826 & 3,21 & 3,62 & 4,09 & 4,06 \\
\hline & & 25 & 117241 & 117342 & 117203 & 2,54 & 117536 & 7,44 & 0,70 & 0,73 & 0,45 \\
\hline & & 50 & 235753 & 235783 & 235943 & 4,96 & 236140 & 17,43 & 0,26 & 0,18 & 0,10 \\
\hline & & 6 & 29300 & 30456 & 28199 & 1,20 & 28213 & 2,30 & 19,59 & 24,26 & 24,20 \\
\hline & 6 & 12 & 54903 & 55153 & 54601 & 2,35 & 55060 & 3,97 & 4,43 & 5,01 & 4,13 \\
\hline & & 25 & 112765 & 112842 & 112632 & 4,24 & 113158 & 8,87 & 1,198 & 1,31 & 0,84 \\
\hline & & 50 & 219399 & 219434 & 219412 & 7,19 & 219605 & 17,68 & 0,25 & 0,25 & 0,16 \\
\hline & & 6 & 22084 & 23239 & 21925 & 0,27 & 22173 & 1,01 & 5,43 & 6,03 & 5,00 \\
\hline & 2 & 12 & 43185 & 43273 & 42957 & 0,54 & 43233 & 2,29 & 0,28 & 0,83 & 0,17 \\
\hline & & 25 & 89427 & 89459 & 89236 & 1,42 & 89450 & 5,65 & 0,08 & 0,20 & 0,06 \\
\hline & & 50 & 176735 & 176745 & 176519 & 2,21 & 176709 & 11 & 0,02 & 0,02 & 0,03 \\
\hline & & 6 & 20256 & 20449 & 20064 & 0,61 & 20419 & 1,49 & 1,98 & 2,96 & 1,17 \\
\hline CASBTA & 4 & 12 & 39957 & 40046 & 39933 & 1,08 & 40076 & 2,90 & 0,55 & 0,61 & 0,26 \\
\hline & & 25 & 80944 & 80981 & 80941 & 1,89 & 80981 & 5,72 & 0,09 & 0,09 & 0,04 \\
\hline & & 50 & 162405 & 162411 & 162403 & 3,92 & 162413 & 12,14 & 0,02 & 0,02 & 0,02 \\
\hline & & 6 & 19728 & 19984 & 19372 & 1,02 & 19972 & 1,97 & 3,11 & 5,00 & 1,85 \\
\hline & 6 & 12 & 38413 & 38494 & 38382 & 1,77 & 38505 & 3,48 & 0,43 & 0,51 & 0,19 \\
\hline & & 25 & 78819 & 78853 & 78808 & 3,17 & 78841 & 7,10 & 0,10 & 0,12 & 0,07 \\
\hline & & 50 & 154711 & 154716 & 154708 & 6,13 & 154721 & 13,71 & 0,02 & 0,02 & 0,01 \\
\hline & & 6 & 34516 & 36406 & 33262 & 0,45 & 34692 & 1,14 & 11,55 & 15,75 & 10,98 \\
\hline & 2 & 12 & 66568 & 67073 & 65591 & 0,84 & 67104 & 2,90 & 2,00 & 3,52 & 1,18 \\
\hline & & 25 & 137907 & 138040 & 136834 & 1,84 & 138268 & 9,24 & 0,68 & 1,47 & 0,42 \\
\hline & & 50 & 271176 & 271203 & 271191 & 4,39 & 271186 & 27,47 & 0,24 & 0,23 & 0,23 \\
\hline & & 6 & 29851 & 31388 & 28630 & 0,73 & 29060 & 1,67 & 13,64 & 18,49 & 16,75 \\
\hline CASATA & 4 & 12 & 58216 & 58566 & 57127 & 1,41 & 57976 & 3,41 & 3,96 & 5,95 & 4,40 \\
\hline & & 25 & 117409 & 117513 & 117377 & 2,60 & 117724 & 7,70 & 0,68 & 0,71 & 0,42 \\
\hline & & 50 & 236069 & 236100 & 235889 & 5,18 & 236487 & 18,83 & 0,28 & 0,36 & 0,10 \\
\hline & & 6 & 29425 & 30618 & 28207 & 1,29 & 28264 & $\begin{array}{l}2,36 \\
\end{array}$ & 20,71 & 25,92 & 25,67 \\
\hline & 6 & 12 & 54979 & 55243 & 54668 & 2,38 & 54998 & 4,05 & 5,47 & 6,07 & 5,43 \\
\hline & & 25 & 112879 & 112941 & 112751 & 4,29 & 113293 & 9,07 & 1,27 & 1,39 & 0,90 \\
\hline & & 50 & 219561 & 219595 & 219572 & 7,34 & 219781 & 18,11 & 0,28 & 0,28 & 0,18 \\
\hline
\end{tabular}

Tabela 3: Comparação entre limites inferiores para 18 períodos e capacidade apertada

que os autores analisam os limites inferiores do problema de dimensionamento de lotes com uma máquina utilizando a relaxação Lagrangiana. Em [6] é proposta uma 
reformulação para o problema utilizando a ideia de redefinição de variáveis proposta em [5] e as restrições de demanda do problema são relaxadas, aplicando assim uma decomposição por períodos ao invés da decomposição clássica por itens. Os autores encontram limites inferiores melhores comparados com a decomposição clássica. Outros autores, tais como, [2] e [8] também analisam procedimentos semelhantes para o caso de única máquina.

A ideia foi estendida, no presente trabalho, considerando um ambiente com máquinas paralelas. Foi utilizada a mesma ideia de redefinição de variáveis e foi proposta uma reformulação para o problema com máquinas paralelas. Os limites inferiores foram gerados utilizando a decomposição por períodos e por máquinas e comparados com os limites inferiores encontrados em [10] em que a decomposição clássica por ítens é utilizada.

Os limitantes foram testados para diferentes tipos de problemas e os resultados foram comparados utilizando como limite superior, soluções encontradas pelo pacote de otimização CPLEX 12.2. O método proposto encontrou em praticamente todos os testes limites inferiores melhores do que os gerados pela decomposição clássica proposta em [10], apesar dos tempos computacionais serem maiores devido a notável diferença entre a dificuldade de resolução dos subproblemas. Além disso, o método proposto se mostrou competitivo comparado ao CPLEX, uma vez que na maioria das instâncias obteve desempenho superior ao solver mesmo após as ramificações, o que ocorre principalmente nas instâncias maiores com 25 e 50 itens.

Tendo em vista estender o trabalho, bem como melhorar os resultados computacionais tem-se como próximo passo a melhoria da heurística desenvolvida neste trabalho. Ideias clássicas da literatura que envolvem a transferência de lotes podem ser utilizadas, tais como a heurística de eliminação de lotes proposta em [3].

\title{
Agradecimentos
}

Os autores agradecem a professora Franklina M.B. Toledo, por fornecer o código computacional de seu método, bem como, as instâncias utilizadas; agradecem também as contribuições feitas pelos árbitros anônimos da revista TEMA, que em muito melhoraram a versão deste trabalho.

\begin{abstract}
This paper addresses the single stage lot-sizing problem in parallel machines. Each item can be produced on any machine, and incurs a setup time before to start the production. The objective of this paper is to obtain lower bounds of good quality for this problem. A solution method is developed based on a reformulation of the problem and the Lagrangian relaxation of a set of constraints. Some computational results are presented comparing the proposed method with a method from the literature and with a computational package.
\end{abstract}

\section{Referências}

[1] P.M. Camerini, L. Fratta, F. Maffioli, On improving relaxation methods by modified gradient techniques, Mathematical Programming Study, 3 (1975), 2654. 
[2] W.H. Chen, J.M. Thizy, Analysis of relaxation for the multi- item capacitated lot-sizing problem, Operations Research, 26 (1990), 29-72.

[3] Z. Degraeve, R. Jans, A new dantzig-wolfe reformulation and branch-and-price algorithm for the capacited lot-sizing problem with setup times, Operational Research, 55 (2007), 909-920.

[4] M. Diaby, H. Bahl, M.H. Karwan, S. Ziont, Capacitated lot-sizing and scheduling by lagrangean relaxation, European Journal of Operational Research, 59 (1992), $444-458$.

[5] G.B. Eppen, R.K. Martin, Solving multi-item capacitated lot-sizing problems using variable redefinition, Operations Research, 6 (1987), 832-848.

[6] R. Jans, Z. Degraeve, Improved lower bounds for capacitated lot sizing problem with setup time, Operation Research Letters, 32 (2004), 185-195.

[7] C.M.O. Pimentel, F.P. Alvelos, J.M.V. Carvalho, Comparing Dantzig-Wolfe decompositions and branch-and-price algorithms for the multi-item capacitated lot sizing problem, Optimization Methods and Software, 25 (2010), 229-319.

[8] H. Sural, M. Denizel, L.N. van Wassenhove, Lagrangean relaxation based heuristic for lot sizing with setup times, European Journal of Operational Research, 195 (2009), 51-63.

[9] J.M. Thizy, L.N.V. Wassenhove, Lagrangean relaxation for the multi-item capacitated lot-sizing problem: a heuristic implementation, AIIE Transactions, 17 (1985), 64-74.

[10] F.M.B. Toledo, V.A. Armentano, A Lagrangian-based heuristic for the capacitated lot-sizing problem in parallel machines, European Journal of Operational Research, 175 (2006), 1070-1083. 